\title{
Phase-field Crystals with Elastic Interactions
}

\author{
Peter Stefanovic ${ }^{1}$, Mikko Haataja ${ }^{2}$, Nikolas Provatas ${ }^{1}$ \\ ${ }^{1}$ Department of Materials Science and Engineering and Brockhouse Institute for Materials Research, \\ McMaster University, ${ }^{2}$ Department of Mechanical and Aerospace Engineering and the Princeton \\ Institute for the Science and Technology of Materials (PRISM), Princeton University
}

(Dated: November 13, 2018)

\begin{abstract}
We report on a novel extension of the recent phase-field crystal (PFC) method introduced in [Elder et al., Phys. Rev. Lett., 88, 245701:1-4 (2002)], which incorporates elastic interactions as well as crystal plasticity and diffusive dynamics. In our model, elastic interactions are mediated through wave modes that propagate on time scales many orders of magnitude slower than atomic vibrations but still much faster than diffusive times scales. This allows us to preserve the quintessential advantage of the PFC model: the ability to simulate atomic-scale interactions and dynamics on time scales many orders of magnitude longer than characteristic vibrational time scales. We demonstrate the two different modes of propagation in our model and show that simulations of grain growth and elasto-plastic deformation are consistent with the microstructural properties of nanocrystals.
\end{abstract}

PACS numbers: 46.15.-x, 61.82.Rx, 62.25.+g, 62.30.+d, 63.22.+m

The deformation of a solid triggers processes which operate across several length and time scales. On long length and time scales its behavior can be described by a set of hydrodynamic equations [1, 2], which describe, e.g., elastic deformation dynamics of the body. On atomic length $\left(\sim 10^{-10} \mathrm{~m}\right)$ and time $\left(\sim 10^{-13} \mathrm{~s}\right)$ scales, on the other hand, the dynamics can be captured by direct molecular dynamics (MD) simulations, which incorporate local bonding information either through direct quantum-mechanical calculations or semi-empirical many-body potentials. While innovations in computing methods have greatly improved the efficiency of MD simulations, standard atomistic computer simulations are still limited to fairly small system sizes $\left(\sim 10^{9}\right.$ atoms $)$ and short times $\left(\sim 10^{-8} \mathrm{~s}\right)$. This limitation is most severe when developing simulation models to study the physics and mechanics of nanostructured materials, where the relevant length scales are atomic and time scales are mesoscopic. In this regime, the available numerical tools are rare.

Progress towards alleviating this limitation has recently been made by the introduction of a new modeling paradigm known as the phase-field crystal (PFC) method 3]. This method introduces a local atomic mass density field $\rho(\mathbf{r})$ in which atomic vibrations have been integrated out up to diffusive time scales. Dissipative dynamics are then constructed to govern the temporal evolution of $\rho$. Unfortunately, the original PFC model evolves mass density only on diffusive time scales. In particular, it does not contain a mechanism for simulating elastic interactions, an important aspect for studying, for example, the deformation properties of nanocrystalline solids.

In this Letter, we introduce a modified phase-field crystal (MPFC) model that includes both diffusive dynamics and elastic interactions. This is achieved by exploiting the separation of time scales that exists between diffusive and elastic relaxation processes in solids. In particular, the MPFC model is constructed to transmit long wave- length density fluctuations with wave modes that propagate up to a time scale $t_{w}$, after which the strain-relaxed density field continues to evolve according to diffusive dynamics. The key feature of our approach is that the value of $t_{w}$ can be chosen to be much smaller than the characteristic time scale of diffusion and still much larger than $1 / \omega_{D} \sim 10^{-13}$ s, where $\omega_{D}$ denotes the Debye frequency.

The phase field crystal methodology begins by introducing an effective free-energy expanded to lowest order in the mass density $\rho(\mathbf{r})$ :

$$
F[\rho ; T]=\int\left(\rho / 2\left[r+\left(q_{o}+\nabla^{2}\right)^{2}\right] \rho+\rho^{4} / 4\right) d^{2} x,
$$

Here, $r \sim\left(T-T_{m}\right) / L c_{P}$ and $T_{m}, L$ and $c_{P}$ are, respectively, the melting temperature, the latent heat of fusion and specific heat at constant pressure of the pure material. Also, $q_{o}=2 \pi / a$, where $a$ is the equilibrium lattice spacing. This free energy is identical to the one used in Ref. 3], and gives rise to a phase diagram of coexisting liquid, solid, and striped phases, as shown in Fig. 1(a). In the solid phase, $\rho$ is non-zero everywhere and spatially periodic on the atomic scale with hexagonal symmetry in two spatial dimensions. In the liquid phase, $\rho$ takes on a constant value everywhere. In the PFC formalism lattice sites are always occupied and vacancy diffusion and topological defects are represented via modulations of the local density amplitude and wavelength. Elastic constants can be determined by computing $C_{i j k l}=\delta^{2} F / \delta u_{i j} \delta u_{k l}$, where $u_{i j}$ represents the strain of a particular deformation state.

In the original PFC model, the evolution of the mass density is given by

$$
\partial \rho / \partial t=\alpha^{2} \nabla^{2}(\delta F[\rho ; T] / \delta \rho) .
$$

where $\alpha$ is a constant. A severe limitation of the PFC model in Eq.(2) is that it only allows for diffusive density relaxation. The model does not inherently contain a 


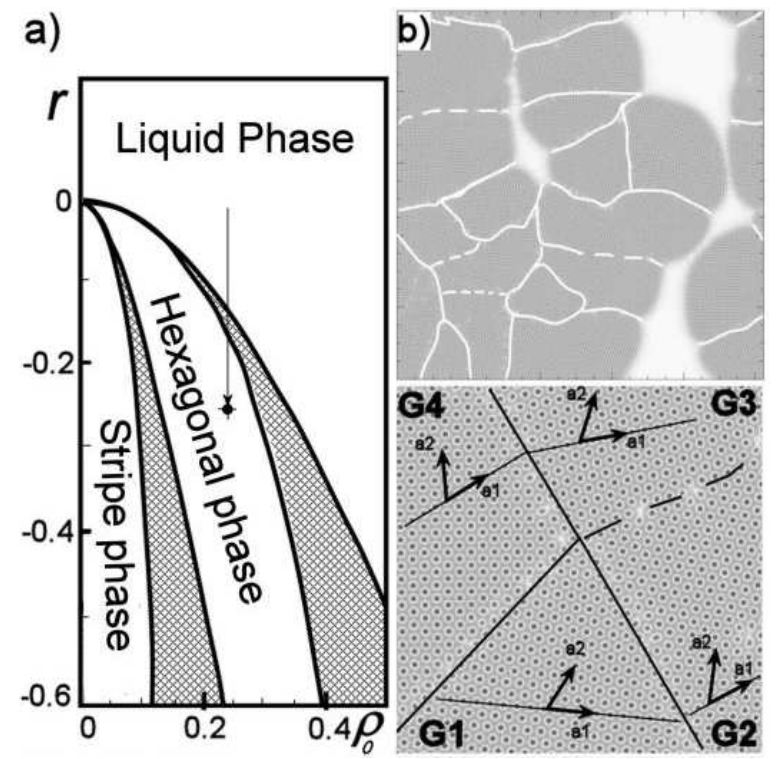

FIG. 1: (a) Phase diagram indicating isothermal quench. The diagram is symmetric around $\rho_{0}=0$. (b-top) Snapshot in the evolution of polycrystalline solidification using the MPFC model. Grain boundaries are highlighted in white. (bbottom) Zoom-in of 4 crystal grains and their orientations.

suitable separation of times scales between phase transformation kinetics and the much more rapid ("instantaneous") elastic relaxation. This precludes the study of phase transformation phenomena in the presence of complex mechanical deformations. It should be pointed out that while homogeneous deformations can be imposed through an affine transformation [5], this method is inapplicable in cases where non-homogeneous stress distributions arise. As will be demonstrated below, these serious shortcomings of the original PFC model can be circumvented in a way that allows us to preserve the quintessential advantage of the PFC model, namely, the ability to simulate atomic-scale interactions and dynamics on time scales many orders of magnitude longer than molecular dynamics time scales. Most importantly, our modified model naturally incorporates "instantaneous" elastic interactions.

We begin by introducing a modified PFC equation given by

$$
\frac{\partial^{2} \rho}{\partial t^{2}}+\beta \frac{\partial \rho}{\partial t}=\alpha^{2} \nabla^{2} \mu+\xi
$$

where $\mu=\delta F[\rho ; T] / \delta \rho$, while $\beta$ and $\alpha$ are phenomenological constants. $F$ is the free-energy introduced in Eq. 1 and $\xi$ is a Gaussian random variable with correlations satisfying $\left\langle\xi \xi^{\prime}\right\rangle=k_{B} T \beta q_{o}^{d-4} / \lambda^{2} \nabla^{2} \delta\left(\vec{x}-\overrightarrow{x^{\prime}}\right) \delta\left(t-t^{\prime}\right)$. Henceforth, we will set $\xi=0$. Equation 3 is of the form of a damped wave equation, containing two propagating density modes at early time and one diffusive mode at late times. Specifically, the fast dynamics of the MPFC model are governed by the first term of Eq.(3), while the late time dynamics are governed by Eq.(2).

To elucidate the dynamics described by Eq. (3), we performed a Floquet stability analysis. This was done by assuming a perturbation in the density of the form $\rho_{p}=$ $\rho_{e q}+\delta \rho$, where $\rho_{e q}=\rho_{o}+\sum_{n, m} a_{n, m} e^{i \vec{G}_{n, m} \cdot \vec{r}}$, with $\rho_{o}$ the average density, $\vec{G}_{n, m}=n \hat{x}+(n+2 m) / \sqrt{3} \hat{y}$ the triangular reciprocal lattice vectors and $a_{n, m}$ their corresponding amplitudes. Here, $\delta \rho=\sum_{n, m} b_{n, m}(t) e^{i \vec{G}_{n, m} \cdot \vec{r}+i \vec{Q} \cdot \vec{r}}$, where $\vec{Q}$ is a perturbation wave vector and $b_{n, m}(t)$ the perturbation amplitude of mode $(m, n)$. Substituting $\rho_{p}$ into the model and expanding to linear order gives an equation for $b_{n, m}$. The leading order mode satisfies $b_{0,0} \sim e^{i \omega t}$. The dispersion relation $\omega(Q)$ is given by $\omega(Q)=i \beta / 2 \pm \Lambda(Q) / 2$, where $\Lambda(Q)=$ $\sqrt{-\beta^{2}+4 \alpha^{2} Q^{2}\left[3 \rho_{o}^{2}+r+\left(Q^{2}-q_{o}^{2}\right)^{2}+9 / 8 A_{\text {min }}^{2}\right]}$. Note that when $4 \alpha^{2} Q^{2}\left[3 \rho_{o}^{2}+r+\left(Q^{2}-q_{o}^{2}\right)^{2}+9 / 8 A_{\text {min }}^{2}\right] \gg$ $\beta^{2}$, the dispersion is approximately $\omega(Q) \approx i \beta / 2 \pm$ $2 \alpha Q \sqrt{3 \rho_{o}^{2}+r+\left(Q^{2}-q_{o}^{2}\right)^{2}+9 / 8 A_{\text {min }}^{2}} \equiv i \beta / 2 \pm v_{\text {eff }} Q$. This dispersion describes a pair of waves that propagate undamped for time $t_{w} \approx 2 \beta^{-1}$ and distance $L \sim v_{\text {eff }} t_{w}$, after which they become effectively diffusive as in Ref. [3]. It is precisely these propagating modes which mediate elastic interactions in the model. Details of this calculation as will be presented elsewhere [6].

This analysis demonstrates that Eq.(3) allows us to transmit disturbances across long distances using wave modes that propagate on a time scale $t_{w}$. This allows all atomic positions to relax to a position close to their deformed equilibrium positions prior to any significant diffusion taking place. Most notably, the time scale $t_{w}$ can be set many orders of magnitude larger than characteristic vibrational time scales $\left(\sim 10^{-13}\right.$ s) but still significantly faster than the scale on which diffusive processes occur.

We now turn to the treatment of the fully non-linear evolution of Eq. (3). The details of our numerical procedures are as follows. All simulations were conducted on a rectangular grid using periodic boundary conditions. Space was measured in units of the lattice constant $a$, while the grid size $\Delta x$, time step $\Delta t$ and coefficients $\alpha, \beta$ were chosen according to the particular application. External loads were applied to the boundary of our system by using a penalty function method. In this method, an additional term, of the form $P=M(x, y, t) \sqrt{\left(\rho-\rho_{b d y}\right)^{2}}$, is added to the free energy. This term couples the sample density $\rho$ to an imposed periodic density field $\rho_{\mathrm{bdy}}$. The support of $\rho_{\mathrm{bdy}}$ is the same as the support of the function $M(x, y, t)>0$, which defines the shape of the desired loading surface. The form of $P$ thus couples some portion of the sample's density (e.g. near the sample boundaries) to the imposed boundary potential $\rho_{b d y}$, which results in the sample's density field becoming slaved to the peaks of $\rho_{\text {bdy }}$ as $|M(x, y, t)| \rightarrow \infty$. As the applied potential field is translated, the sample's density field, along the load- 
ing surfaces, adiabatically follows the applied field. This specific form of $P$ also assures that our penalty function does not alter the equilibrium phase diagram of the basic free energy functional $F[\rho ; T]$ defined above.

We first simulated isothermal solidification using Eq.(3) by preparing the system in the liquid state and subsequently setting the temperature below the coexistence line in the phase diagram. In this case, the dynamics gives rise to nucleation and growth of the solid phase in the presence of thermal fluctuations. To facilitate nucleation, several nucleation sites were initiated in the metastable liquid phase in the form of random (Gaussian) fluctuations. During solidification, we found that the effect of the first term in Eq.(3) was negligible, and the growth rates and morphology were essentially indistinguishable from those using Eq.(2). Figure 11 illustrates growth and impingement of several nuclei in an undercooled melt. The simulation was started with the liquid of average density $\rho_{0}=0.285$ and dimensionless temperature $r=-0.25$; other parameters were set to $(\Delta x, \Delta t, \alpha, \beta)=(\pi / 8,0.001,15,0.9)$. The measured grain boundary energies per unit length are consistent with the usual Read-Shockley form [4, 7]

To demonstrate the presence of elastic relaxation modes in the MPFC model, we performed simulations of an effectively one dimensional single-crystal specimen under uniaxial tension. The system was prepared in the coexistence region as given by the phase diagram, and the solid sample was surrounded by liquid. Model parameters used were $(r, \psi, \Delta x, \Delta t, \alpha, \beta)=$ $(-0.4,0.31, \pi / 8,0.001,15,0.9)$. A tensile load applied to a semi-infinite continuum elastic bar can be theoretically modelled as array of coupled masses and springs along the $x$-axis, as illustrated in Fig. 2. When an atom at the boundary is displaced by an amount $D_{1}$ to the left, a tensile stress wave will propagate to the right. When atomic oscillations stop, a linear displacement distribution, $D(x)=D_{1} x / L$, will be established along the bar. Plots of displacement vs. position in the case of constant strain rate applied to the boundary atom are shown in Fig. 3 at three different times. Here, the displacements were extracted by a peak tracking method, where the locations of local maxima in $\rho$ were tabulated after each time step. The data clearly shows that the response of the system is consistent with elasticity theory.

To make contact with the previous PFC formulation in Eq.(2) [4], we repeated the same simulations with a ten-fold increase in the damping parameter $\beta=9$. The computed displacements, plotted in the inset of Fig. 3 . show that the response becomes viscoelastic as damping is increased. Therefore, Eq.(2) alone does not adequately describe elastic responses in strained crystals at finite strain rates, while Eq.(3) naturally incorporates such phenomena. We note that for simple modes of deformation, Eq.(2) can still be used to model elastic relaxation by uniformly adjusting all the atomic peaks of the

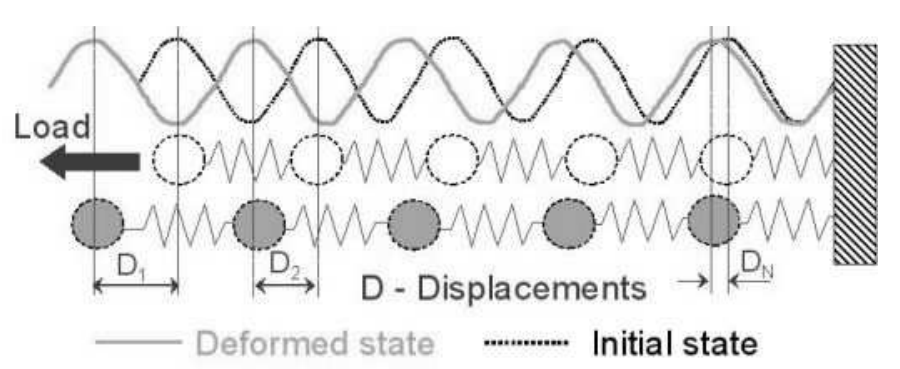

FIG. 2: Schematic illustration of the atomic locations before and after tension is applied in the MPFC model.

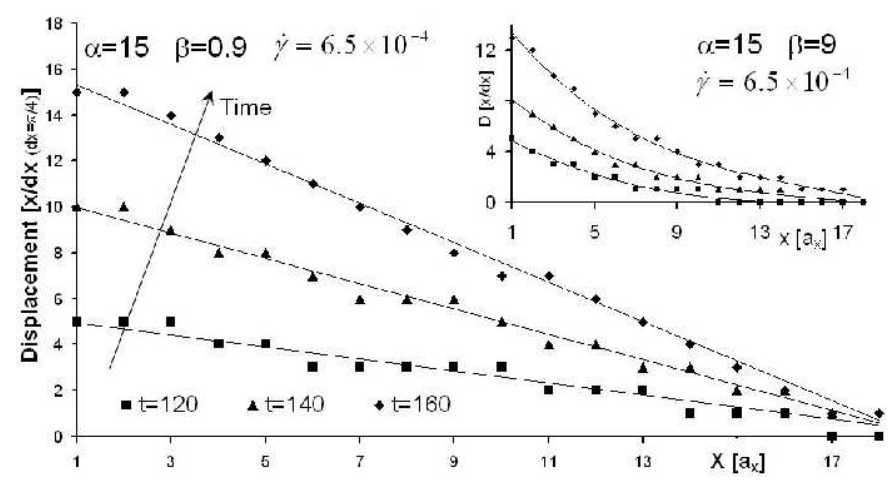

FIG. 3: The displacements along a one-dimensional sample in simple uniaxial tension at three different times (top). Linear profiles are consistent with linear elasticity theory. Inset: a ten-fold increase in $\beta$ leads to visco-elastic behavior.

density field $\rho$ after a specified number of numerical time steps, which is equivalent to carrying out an affine transformation. This method is applicable in, e.g., elucidating the glide dynamics of a single dislocation [5]. However, this approach cannot be used to handle elastic relaxation in the case of complex geometries, non-uniform stresses, and high strain rates [8, 9, 10, 11].

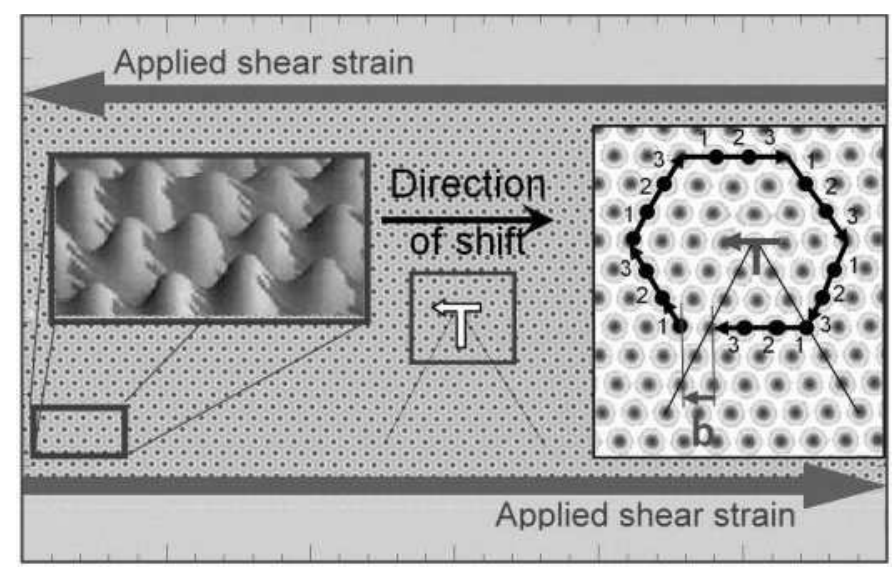

FIG. 4: A portion of the sample used to examine dislocation glide velocity. Parameters used: $(r, \psi, \Delta x, \Delta t, \alpha, \beta)=$ $(-1,0.49, \pi / 4,0.001,15,0.9)$. 


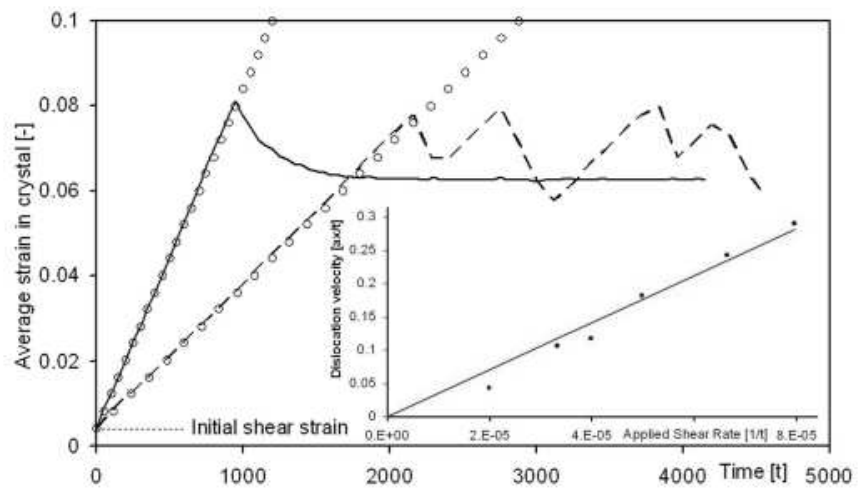

FIG. 5: Two regimes of dislocation glide. For high strain rates we observed continuous glide, while at lower strain rate the dislocation set into a stick-slip motion. Inset: Dislocation glide velocity Vs. applied strain rate.

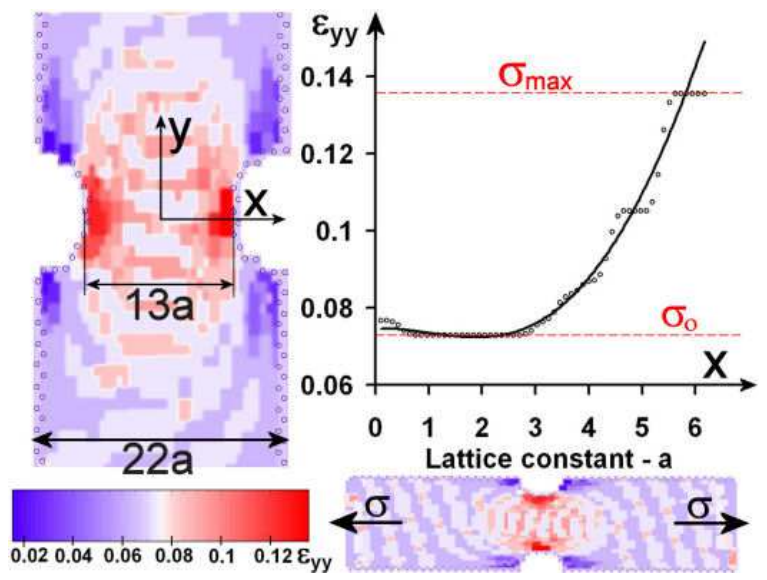

FIG. 6: Strain concentration in a double notched sample under a uniaxial tension. Left: A strain map of the center portion of the sample displayed at the bottom. Boundary atoms are highlighted in black. Right: Plot represents a strain profile from the center of the sample into the root of the notch. The solid line is a guide to the eye.

We also examined the dynamics of individual dislocations. The set-up for these simulations are shown in Fig. 4 Specifically, the top part of the crystal initially contains $\mathrm{N}$ atoms and the bottom part $\mathrm{N}+1$. After the sample equilibrated an edge dislocation formed and a constant shear strain rate was applied. The time-averaged dislocation glide velocity $\bar{v}$ was found to be a linear function of the strain rate $\dot{\gamma}$, consistent with classical dislocation theory. This theory predicts that $\bar{v}=\dot{\gamma} /\left(\rho_{d} b\right)$, where $\rho_{d}$ is the dislocation density and $b$ is the magnitude of the Burger's vector 12 .

In order to elucidate the local dynamics of individual dislocations, we computed the average strain in the crystal as a function of time for different strain rates. These results, shown in Fig. 5 revealed two regimes of dislocation glide. The first was characterized by continuous glide (observed at large $\dot{\gamma}$ ) and the second by a stickslip gliding of the dislocation at low $\dot{\gamma}$. In both cases the applied plastic strain was relieved by the motion of the dislocation, and the time-averaged strain remained constant.

To further illustrate the properties of our MPFC model, the effect of uniaxial tension in a notched sample was examined. Figure [6] shows that strain (stress) in a notched sample appropriately concentrates near the notches, as expected from linear elasticity theory. In particular, treating the case of a double notched plate the stress concentration for this geometry is $K_{t}=$ $\sigma_{y y}^{\max } / \sigma_{y y}^{0}=1.8$ [13], which is in excellent agreement with our simulation result 1.81 . It is noteworthy that a simulation with the PFC model (Eq. 2) for the same system and using an affine transformation to approximate the strains in the sample, failed to produce the expected strain concentration.

In conclusion, we have introduced a novel phasefield crystal model (MPFC), which extends the previous phase-field crystal formalism by generating dynamics on two time scales. Atomic positions are relaxed rapidly at early times in a manner consistent with elasticity theory, while late time dynamics are governed by diffusive dynamics characteristic of phase transformation kinetics, vacancy diffusion, grain boundary kinetics and dislocation climb. It is expected that the MPFC model will help open a new window into the study of phase transformation kinetics and microstructure heterogeneity in high strain rate loading of nanocrystalline solids.

This work has been in part supported by the National Science and Engineering Research Council of Canada (NP) and an NSF-DMR Grant No. 0449184 (MH).

[1] C. P. Enz, Rev. of Mod. Phys., 46, 705 (1974).

[2] P. Fleming III and C. Cohen, Phys. Rev. B, 13, 500 (1976).

[3] K. R. Elder, M. Katakowski, M. Haataja, and M. Grant, Phys. Rev. Lett., 88, 245701 (2002).

[4] K. R. Elder, M. Grant, Phys. Rev. E, 70, 051605 (2004).

[5] J. Berry, K. R. Elder and M. Grant, cond-mat(0510759)

[6] P. Stefanovic, M. Haataja, N. Provatas, to appear (2006).

[7] J. A. Warren, R. Kobayashi, A. E. Lobkovsky, and W. C. Carter, Acta Mater., 51, 6035 (2003).

[8] J. Schiotz and K. Jacobsen, Science, 301, 1357 (2003).

[9] H. VanSwygenhoven, M. Spaczer, A. Caro, D. Farkas, Phys. Rev. B, 60, 22 (1999).

[10] V. Yamakov and et al., Nature Materials, 1, 1 (2002).

[11] B. Remington, Met. Mat. Trans. A, 35, 2587 (2004).

[12] W. G. Johnston and J. J. Gilman, J. Appl. Phys., 30, 129 (1959).

[13] R.E. Peterson, Stress concentration design factors, Wiley and Sons., Inc., 1953. 